\title{
Clinical study of the safety and effectiveness of a novel over-the-counter bleaching tray system
}

This article was published in the following Dove Press journal:

Clinical, Cosmetic and Investigational Dentistry

2I February 20I4

Number of times this article has been viewed

\section{K Michael Ghalili' \\ Kamal Khawaled ${ }^{2}$ \\ Doran Rozen² \\ Veda Afsahi ${ }^{3}$}

'Department of Prosthodontics, New York University College of Dentistry, New York, NY, USA; ${ }^{2}$ Department of R\&D; Department of Clinical Affairs, Syneron Beauty, Ltd, Yokneam, Israel; ${ }^{3}$ Veda Faith Afsahi Inc., Fountain Valley, CA, USA
Correspondence: K Michael Ghalili 65 West 55th Street, Suite 305, New York, NY 10019, USA

$\mathrm{Tel}+\mathrm{I} 21258 \mid 5360$

Email kghalili@gmail.com
Abstract: We investigated color change, gingival irritation, and tooth sensitivity in patients undergoing at-home vital tooth bleaching with a novel over-the-counter bleaching tray system. Tooth color shade in anterior teeth, supragingival plaque and gingivitis in Ramfjord teeth, as well as visual assessment of teeth gingival tissues and mucosa were evaluated in-office prior to treatment, after two consecutive applications of the $9 \%$ hydrogen peroxide bleaching product, after eight applications (10 minutes/day for 3 days at home), and after ten applications (50 minutes exposure over 5 days). Color stability was evaluated at 3 months after completing the treatment regimen. Over-the-counter bleaching products can be used by the patient at home without dentist supervision, but are frequently associated with gingival irritation and tooth sensitivity despite low concentrations of peroxide agents. Our investigations showed that the treatment is tolerable and safe with a low incidence of adverse effects. Any adverse effects associated with use of the whitening gel and tray are temporary, easily controlled, and often disappear within minutes of treatment. Statistical analysis revealed significant improvement in teeth whitening following treatment (mean color change of seven shades) and at three months after treatment.

Keywords: at home tooth-whitening, hydrogen peroxide teeth whitening, over-the-counter tooth-whitening, teeth bleaching

\section{Introduction}

As a person ages, the adult teeth often become darker, losing their natural white due to changes in enamel and dentin properties. Extrinsic staining by bacterial byproducts, food pigments, tobacco, and dark drinks, such as coffee, tea, and red wine, can also influence tooth discoloration. With increased awareness of oral aesthetics, many individuals are looking to enhance their appearance through "whiter" teeth rather than simply seeking restorative treatments. ${ }^{1,2}$

Professional in-office bleaching procedures can produce significant improvement in the cosmetic appearance of most stained teeth; however, this method may result in local side effects on the oral mucosa and dental tissues during whitening, such as pulp sensitivity, oral mucosa burns, and alteration of enamel surface. ${ }^{3}$ Most of the local effects result from the high concentration of hydrogen peroxide in the product used and the technique of application during repeated treatments.

Supervised bleaching with custom trays is the most common modality for at-home vital tooth bleaching, though both mineral loss and tooth sensitivity are still common problems. ${ }^{4,5}$ Furthermore, such home bleaching protocols require overnight treatment for up to several weeks. 
We investigated a novel commercially-available over-the-counter bleaching system with a flexible doublesided dental tray that uses low-energy current to activate the peroxide-containing bleaching agent. We proposed that shorter exposure to the peroxide-containing gel compared with overnight dental trays, as well as reduced contact with gingiva due to tray design compared with whitening strips, might reduce sensitivity and adverse effects.

\section{Methods and materials Patients}

Forty-three patients were selected using the following inclusion criteria: 18 to 65 years old and in good health; needed to have all maxillary and mandibular anterior teeth without restorations or caries; color A3 in at least two maxillary and mandibular anterior teeth (not including canines); and to agree to return for scheduled visits and follow-up examinations. The exclusion criteria were: orthodontic appliances, or full or partial dentures; gingivitis or periodontitis; any known reaction to peroxides; use of any bleaching agents within the last year; pregnant or lactating women; and tetracycline stained teeth. This research was approved by a private investigational review board, and all participants provided written informed consent.

\section{Assessments}

Measurements were performed by the study investigators (KMG and VA) at their clinics during in-office visits on day 1 and day 5 of treatment. Tooth color was also measured at 3 months after completing the 5-day treatment regimen. The same investigator performed both baseline and posttreatment measurements for the study participants enrolled at their clinics.

\section{Tooth shade grade}

Tooth color shade was evaluated using an established and standardized scale of 16 shades: VITA classical shade guide (Vident, Brea, CA, USA). Study investigators, experienced in using the VITA shade guide for cosmetic dentistry, evaluated tooth shade of the facial surfaces of the four maxillary anterior and four mandibular anterior teeth (without canines). Color measurements were performed under standardized conditions and lighting.

\section{Gingival measurement/safety}

In accordance with American Dental Association guidelines, gingival health was measured using the Gingival and the Plaque Indices (Löe). ${ }^{6}$ For the purpose of this study, supragingival plaque and gingivitis were evaluated in Ramfjord teeth only (number 3, 9, 12, 19, 25, and 28). The investigators recorded readings at each evaluation period.

\section{Oral soft tissue exam}

Gingival, buccal, and labial mucosa, as well as tongue and hard palate, were examined and any abnormal findings were reported.

\section{Tooth sensitivity measurement}

Subject self-assessment of perceived tooth sensitivity was reported using a 5-point Likert Scale: $0=$ no pain to $4=$ severe (excruciating, constant pain).

\section{Treatments}

Subjects were provided with the at-home bleaching system (Pearl Brilliant White Ionic Teeth Whitening System; Syneron Beauty Ltd., Yokneam, Israel), consisting of a flexible silicone dental tray with superior and inferior arcs (Figure 1) and 20 ampoules of $9 \%$ hydrogen

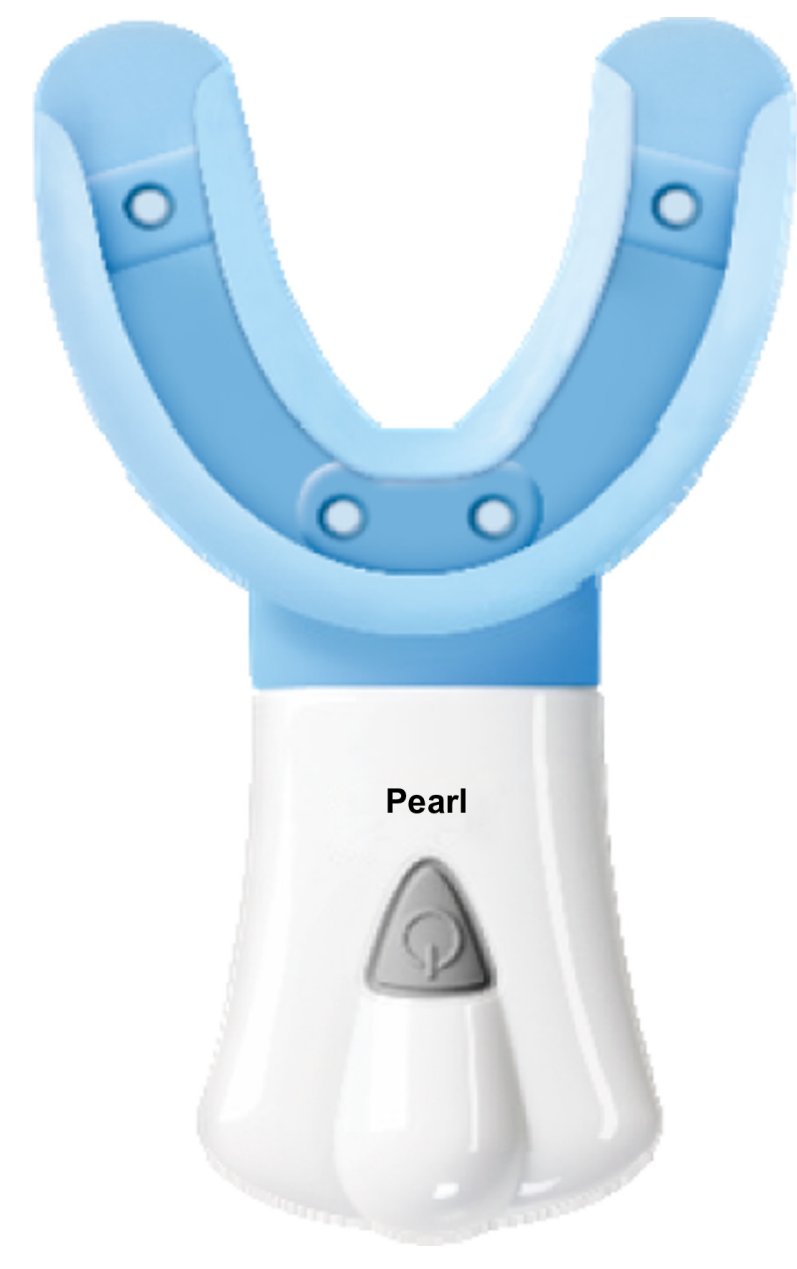

Figure I At-home teeth whitening tray (superior arc shown). 
peroxide (HP) gel. Electrodes in the walls of the tray deliver a low-level electrical current (4 to 15 milliamperes) to activate the peroxide-containing gel, which then diffuses through the enamel, causing oxidation and reduction of organic pigments.

After initial photos were taken, and color and oral tissue measurements were done, the mouth tray was given to each patient, as well as four gel-filled ampoules ( $9 \%$ HP) and instructions for use. Subjects self-performed two consecutive 5-minute treatments in the clinic on day 1 . Subjects then took the study equipment home and were instructed to perform two treatments per day at home on days 2, 3, and 4. The final two treatments were performed in the clinic on day 5 (50 minutes total exposure).

\section{Results}

Forty-three subjects (24 females, 19 males; mean age $45 \pm 13$ years) were enrolled in the study and performed day 1 treatments. Nearly $60 \%$ of subjects were Caucasian; remaining subjects were of African-American, Asian, Hispanic, and other ethnicities. Sixty percent of subjects had never smoked, $28 \%$ were previous smokers, and $12 \%$ were light smokers ( $<10$ pack-years). Most subjects were daily coffee/tea drinkers or drank dark-colored beverages. Five subjects did not present for day 5 evaluations due to Hurricane Sandy but were included in the evaluation analysis prior to the day 5 study point; therefore, 38 subjects had evaluable data on day 1 and day 5 .

\section{Baseline measurements}

At baseline, 344 teeth were evaluated in the 43 subjects. The overall average tooth shade was eleven, with a range from two to 16 . Of the 344 teeth, 24 teeth (22 upper and two lower) had a shade grade of less than A3; 320 teeth had a shade grade of A3 or greater and were included in the tooth shade analysis. With the exception of one subject with sublingual tori, normal findings were reported for gingival, buccal, and labial mucosa, as well as for tongue and hard palate. Four subjects reported slight teeth sensitivity associated with mechanical brushing and drinking cold drinks. The majority of the subjects $(79 \%)$ had no plaque present on the teeth at baseline (a plaque index score of zero, defined as excellent) in the Ramfjord teeth. Eight subjects had ratings within the range of $0.1-0.9$ (defined as good - a film of plaque was present on several teeth) and one subject had a rating of one, which is defined as fair. Additionally, $87 \%$ of subjects had normal gingiva (score of zero). Five subjects had mild inflammation, but no bleeding on probing in the buccal and lingual surfaces.

\section{Day I posttreatment assessments}

Following 5 minutes of treatment, teeth discoloration was significantly improved from baseline $(P<0.05$; paired $t$-test $)$ with average shade improvement of 2.3 shades. After two applications, 34 out of 43 (79\%) subjects reported no pain at all associated with treatment, seven subjects reported slight discomfort, and two subjects reported mild discomfort. Upon oral tissue examination following the two treatment applications on day 1 , there were two subjects who experienced blanching in the buccal mucosa. This was temporary and resolved without intervention prior to the subjects leaving the clinic.

\section{Day 5 posttreatment assessments}

Of the 43 subjects enrolled, 38 subjects presented to the clinics on day 5 for final treatments and assessments. Following 50 minutes of treatment, mean tooth shade change was seven shades (range: two to 12 shades; Figure 2). An example of the treatment results is shown in Figure 3.

Following 50 minutes of treatment, $82 \%$ of subjects reported no pain at all, five subjects reported slight discomfort, one subject reported mild discomfort, and one

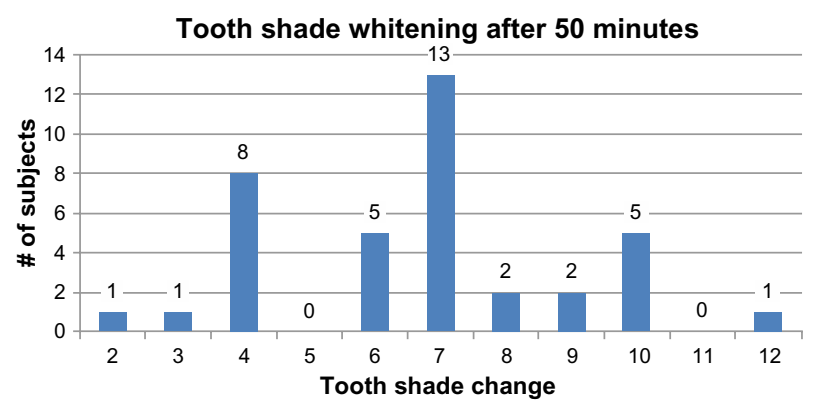

Figure 2 Distribution of color-shade change after 50-minute treatment regimen. Note: Tooth shade measured according to VITA classical shade guide (Vident, Brea, CA, USA).
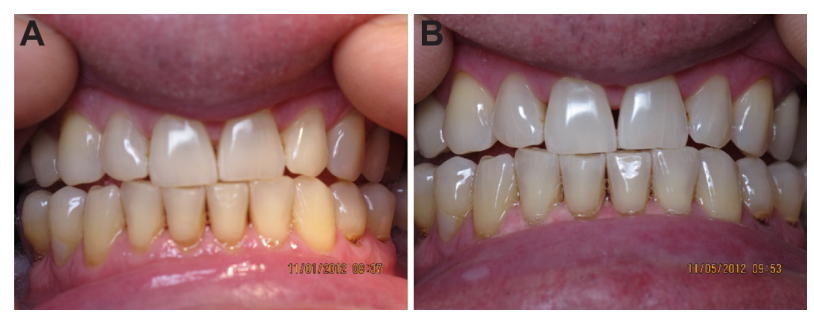

Figure 3 Sixty-two-year-old male.

Notes: (A) A3 tooth grade on the eight anterior teeth. (B) Shade change to A2 following 50 minutes of treatment (average tooth shade change of four shades). 
subject reported moderate discomfort (Figure 4). There was no increase in reports of discomfort associated with treatments performed at home. Furthermore, there were no calls or unanticipated visits to the study investigators during the course of the study.

Of the eight subjects with good plaque scores and one subject with fair plaque score at baseline, there was no plaque present on the teeth following 50 minutes of treatment in five cases, there was a reduction in plaque in two cases, and there was no change following treatment in two cases. The average gingival score for all 38 subjects was 0.05 at baseline (overall gingival index score of $0.1-1=$ good). Following 50 minutes of treatment, there was no additional inflammation due to the bleaching gel and the overall gingival index score was reduced slightly to 0.04 for the 38 subjects evaluated. Furthermore, the 33 subjects with normal gingiva at baseline maintained normal gingiva following treatment.

There were 38 subjects who performed the ten treatment applications following the 5-day protocol. Five subjects performed two treatment applications on day 1 and then dropped from the study. A total of 390 self-treatments were performed over the course of the study. Oral tissue examinations for the 43 subjects on day 1 and 38 subjects on day 5 revealed seven cases of abnormal findings: two subjects experienced mild whitening on the buccal mucosa after two applications (10 minutes exposure); two subject experienced blanching, redness, and sensitivity in the gingival mucosa; one subject experienced blanching in the gingival and buccal mucosa; one subject experienced redness in the papilla area of several upper teeth; and one subject experienced blanching and slight irritation in the hard palate (subject with sublingual tori). All side effects were temporary, often resolving within several minutes of treatment, and did not require intervention.

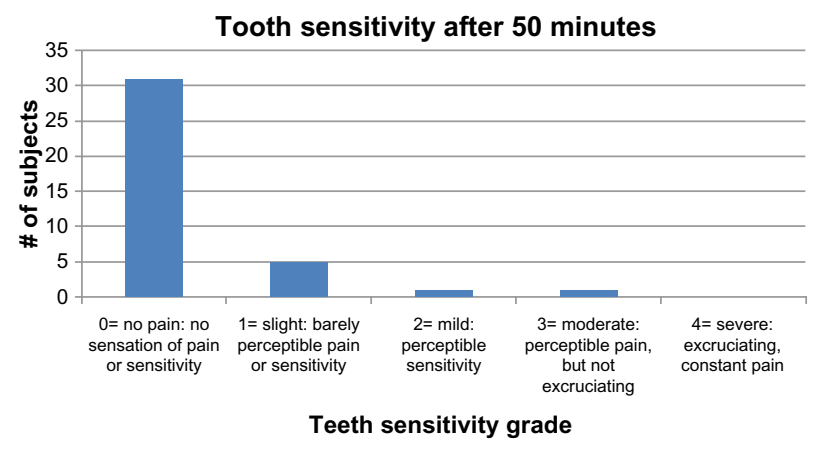

Figure 4 Distribution of sensitivity ratings after 50-minute treatment regimen.

\section{Tooth shade evaluation at 3 months after 5-day treatment protocol}

Of the 38 subjects who performed the day 5 teeth whitening treatments in the clinic, there were 28 subjects available for 3-month follow-up of color-shade assessment. Mean color improvement was 5.7 shades, compared to seven shades after completing the 50-minute protocol. Although paired comparisons of color change after 50-minutes versus after 3 months in the 28 subjects with measurements at both time points revealed a borderline significant difference $(P=0.048$; $t$-test for paired comparisons), it is interesting to note that six subjects actually experienced 0.5 to two shades improvement in color change during follow-up. Increased attention to dental hygiene and desire to maintain an improved oral appearance may have resulted in enhancing the whitening effect of treatment. The average range of shade change remained two to 12 shades of teeth whitening. Measurements correlated with subject experience in that most of the subjects $(75 \% ; 21 / 28)$ reported that they felt that their results were maintained over the 3-month period since treatment.

\section{Discussion}

This study investigated the safety and efficacy of a novel home-use teeth whitening system with $9 \% \mathrm{HP}$, activated by low energy from the electrodes in the tray.

Self-applied bleaching treatments with low levels of carbamide or hydrogen peroxide are widely available to consumers. However, clinical evidence regarding the safety and effectiveness of these products is lacking. Two different studies reported that the prevalence of experienced tooth sensitivity was $50 \%$ for subjects undergoing at-home bleaching procedures. ${ }^{7,8}$ Our clinical investigations have demonstrated a lower level occurrence of side effects of sensitivity and gingival irritation commonly associated with home-use bleaching products. In the current study, subjects were evaluated in-office for sensitivity following the first two consecutive applications (total of 10 minutes exposure) and after the final two applications with $21 \%$ and $18 \%$ of subjects, respectively, experiencing transient sensitivity. The short application time of the bleaching product and its design likely reduces irritation that is associated with the longer exposure required with other bleaching products, or irritation due to contact with gingiva as with whitening strips. Subjects who experienced mild sensitivity were advised to perform daily treatments with one treatment in the morning and one treatment at night at home to prevent sensitivity. Tooth sensitivity tended to decrease as treatment progressed. Addition of potassium nitrate to the hydrogen 
peroxide gel formula likely further reduces sensitivity and gingival irritation as application of this agent causes these side effects to disappear. ${ }^{2}$

In conclusion, the Food and Drug Administration-cleared (K122725) over-the-counter device evaluated in this clinical study uses low-energy current rather than heat or laser as the catalyst for chemical oxidation and, as such, does not produce a significant increase in intrapulpal temperature. ${ }^{9}$ The low heating to the teeth and the potassium nitrate may help to reduce teeth sensitivity. There were no reports of sensitivity to the teeth in the current study. However, there were reports of gum sensitivity in some users. Individuals who experience gum sensitivity due to mechanical brushing or with exposure to cold drinks should consult with a dentist prior to performing at-home bleaching, or use a teeth whitening product with a lower concentration of the bleaching agent. While severely discolored teeth or difficult bleaching cases should be treated initially in the office, we conclude that the at-home bleaching system evaluated in our study produces significant color shade improvement without sensitivity and gingival irritation in the majority of users. Any adverse effects that may occur in some cases with use of the whitening gel and tray are temporary, and easily controlled. User comfort and short-term commitment of wearing the tray for 5-minute applications also provide valuable advantages for patients seeking home-use bleaching alternatives.

\section{Disclosure}

Study equipment was provided by Syneron Beauty, Ltd. Kamal Khawaled and Doran Rozen are employees of Syneron Beauty, Ltd. The authors report no other conflicts of interest in this work.

\section{References}

1. Pretty IA, Edgar WM, Higham SM. The use of QLF to quantify in vitro whitening in a product testing model. Br Dent J. 2001; 191(10):566-569.

2. Demarco FF, Meireles SS, Masotti AS. Over-the-counter whitening agents: a concise review. Braz Oral Res. 2009;23 Suppl 1:64-70.

3. Goldberg M, Grootveld M, Lynch E. Undesirable and adverse effects of tooth-whitening products: a review. Clin Oral Investig. 2010;14(1):1-10.

4. Demarco FF, Meireles SS, Sarmento HR, Dantas RV, Botero T, Tarquinio SB. Erosion and abrasion on dental structures undergoing at-home bleaching. Clin Cosmet Investig Dent. 2011;3:45-52.

5. Borges BC, de Vasconselos AA, Cunha AG, Pinheiro FH, Machado CT, dos Santos AJ. Preliminary clinical reports of a novel nightguard tooth bleaching technique modified by casein phosphopeptideamorphous calcium phosphate (CCP-ACP). Eur J Esthet Dent 2011;6(4):446-453

6. Löe H. The Gingival Index, the Plaque Index and the Retention Index Systems. J Periodontol. 1967;38(6):Suppl:610-616.

7. Bruzell EM, Pallesen U, Thoresen NR, Wallman C, Dahl JE. Side effects of external tooth bleaching: a multi-centre practice-based prospective study. Br Dent J. 2013;215(9):E17.

8. Jorgensen MG, Carroll WB. Incidence of tooth sensitivity after home whitening treatment. J Am Dent Assoc. 2002;133(8):1076-1082.

9. Buchalla W, Attin T. External bleaching therapy with activation by heat, light or laser - a systematic review. Dent Mater. 2007;23(5): 586-596.
Clinical, Cosmetic and Investigational Dentistry

\section{Publish your work in this journal}

Clinical, Cosmetic and Investigational Dentistry is an international, peer-reviewed, open access, online journal focusing on the latest clinical and experimental research in dentistry with specific emphasis on cosmetic interventions. Innovative developments in dental materials, techniques and devices that improve outcomes and patient satisfaction

\section{Dovepress}

and preference will be highlighted. The manuscript management system is completely online and includes a very quick and fair peerreview system, which is all easy to use. Visit http://www.dovepress. com/testimonials.php to read real quotes from published authors. 